\title{
Inhomogeneous Charge Distribution and Superconductivity in the Boson-Fermion Model
}

\author{
Ż. ŚLEDŹ AND M. MierZEJEWSKI \\ Institute of Physics, University of Silesia \\ Uniwersytecka 4, 40-007 Katowice, Poland
}

\begin{abstract}
We analyze a boson-fermion model that accounts for the electrostatic potential of the out-of-plane dopant atoms. We investigate whether this approach may explain recent experimental results obtained for the Bi-based high-temperature superconductors. We demonstrate that in contradistinction to purely fermionic models, even weak electrostatic potential of the dopant atoms may be responsible for a significant modulation of the superconducting order parameter.
\end{abstract}

PACS numbers: 74.20.-z, 74.62.Dh

\section{Introduction}

Scanning tunneling spectroscopy has recently revealed a strong spatial modulation of the superconducting gap in Bi-based cuprates $[1,2]$. In the vicinity of the dopant oxygen atoms the magnitude of the energy gap is enhanced, whereas the height of coherence peaks is strongly reduced [3]. On the other hand, the root mean square of the electron density is smaller than expected. Since its discovery, the inhomogeneity has commonly been attributed to the poorly screened electrostatic potential of the out-of-plane dopant oxygen atoms. We analyze whether these experimental results can be explained within the phenomenological bosonfermion model $[4,5]$, that properly describes many properties of the cuprate superconductors $[6,7]$. In particular, we discuss the profiles of the superconducting order parameters as well as the spatial distribution of fermions and bosons. It has previously been shown that a shift of the bosonic level may substantially modify the strength of the effective pairing between fermions [8]. Consequently, we expect that even weak electrostatic potential of the dopant atoms may be a source of a significant modulation of the superconducting gap. 


\section{Model and details of calculations}

We investigate the two-dimensional boson-fermion model

$$
\begin{aligned}
H= & -\sum_{\langle i, j\rangle \sigma}\left[t_{i j}+\delta_{i j}\left(\mu-U_{i}\right)\right] c_{i \sigma}^{\dagger} c_{j \sigma}+\sum_{\langle i, j\rangle}\left[\omega_{i j}+\delta_{i j}\left(E_{\mathrm{B}}+2 U_{i}\right)\right] b_{i}^{\dagger} b_{j} \\
& +V \sum_{i}\left(b_{i}^{\dagger} c_{i \downarrow} c_{i \uparrow}+b_{i} c_{i \uparrow}^{\dagger} c_{i \downarrow}^{\dagger}\right),
\end{aligned}
$$

where $c_{i \sigma}^{\dagger}$ creates a fermion with spin $\sigma$ at site $i$ and the bosonic operator $b_{i}^{\dagger}$ creates a pair of tightly bound fermions. $E_{\mathrm{B}}=\Delta_{\mathrm{B}}-2 \mu$, where $\Delta_{\mathrm{B}}$ is the bosonic level and $\mu$ is the chemical potential. The charged dopant oxygen atoms, which are placed in the distance $z \simeq 1.5 a$ above the $\mathrm{CuO}_{2}$ planes, shift the fermionic and bosonic energy levels by $U_{i}$ and $2 U_{i}$, respectively. A different shift of these levels originates from the fact that bosons are doubly charged with respect to fermions. We have assumed that the electrostatic potential is screened by charge carriers, which move in the $\mathrm{CuO}_{2}$ planes. Therefore, $U_{i}=U_{0} \sum_{m} \exp \left(-R_{m i} / \lambda\right) / \tilde{R}_{m i}$, where the summation is carried out over all dopant atoms. Here, $U_{0}$ is the impurity potential, $R_{m i}$ is a distance between site $i$ and site $m$ (above which the dopant atom is located) and $\tilde{R}_{m i}=\sqrt{R_{m i}^{2}+z^{2}}$. The boson-fermion interaction has been considered in the mean-field approximation, when the Hamiltonian of the bosonfermion model splits into fermionic and bosonic parts

$$
\begin{aligned}
H^{\mathrm{F}} & =-\sum_{\langle i, j\rangle \sigma}\left[t_{i j}+\delta_{i j}\left(\mu-U_{i}\right)\right] c_{i \sigma}^{\dagger} c_{j \sigma}+\sum_{i} V\left(\rho_{i}^{*} c_{i \downarrow} c_{i \uparrow}+\rho_{i} c_{i \uparrow}^{\dagger} c_{i \downarrow}^{\dagger}\right), \\
H^{\mathrm{B}} & =\sum_{\langle i, j\rangle}\left[\omega_{i j}+\delta_{i j}\left(E_{\mathrm{B}}+2 U_{i}\right)\right] b_{i}^{\dagger} b_{j}+\sum_{i} V\left(\Delta_{i} b_{i}^{\dagger}+\Delta_{i}^{*} b_{i}\right) .
\end{aligned}
$$

$H^{\mathrm{F}}$ and $H^{\mathrm{B}}$ are coupled through the chemical potential and the order parameters $\rho_{i}=\left\langle b_{i}\right\rangle$ and $\Delta_{i}=\left\langle c_{i \downarrow} c_{i \uparrow}\right\rangle$. In the following analysis $\rho_{i}$ will be referred to as the fermionic and $\Delta_{i}$ as the bosonic order parameter since $\rho_{i}$ and $\Delta_{i}$ occur in $H^{\mathrm{F}}$ and $H^{\mathrm{B}}$, respectively.

Fermionic subsystem can be diagonalized with the help of the transformation

$$
c_{i \sigma}=\sum_{n}\left[u_{i n} \gamma_{n \sigma}-s(\sigma) v_{i n}^{*} \gamma_{n \bar{\sigma}}^{\dagger}\right]
$$

where $s(\uparrow)=1, s(\downarrow)=-1$ and $u_{\text {in }}$ and $v_{\text {in }}$ fulfill the Bogoliubov-de Gennes (BdG) equations

$$
\sum_{j}\left(\begin{array}{cc}
\mathcal{H}_{i j} & \delta_{i j} V \rho_{i} \\
\delta_{i j} V \rho_{i}^{*} & -\mathcal{H}_{i j}^{*}
\end{array}\right)\left(\begin{array}{c}
u_{j n} \\
v_{j n}
\end{array}\right)=\varepsilon_{n}\left(\begin{array}{c}
u_{i n} \\
v_{i n}
\end{array}\right)
$$

with $\mathcal{H}_{i j}=-t_{i j}-\delta_{i j}\left(\mu-U_{i}\right)$. Since $\rho_{i}$ explicitly enters Eq. (4), the numerical analysis of the BdG equations should be carried out simultaneously with diagonalization of the bosonic part of the Hamiltonian. We have applied two complementary approaches that allow for a straightforward diagonalization of $H^{\mathrm{B}}$. In the first case, we have restricted our investigations only to localized bosons $\left(\omega_{i j}=0\right)$ but the hard-core effects have been explicitly taken into account. This approach 
will be referred to as a localized boson-fermion (LBF) model. In the second case, we have analyzed mobile bosons neglecting the hard-core effects. This limiting case will be referred to as a mobile boson-fermion (MBF) model. The bosonic subsystem can exactly be diagonalized with the help of a suitable unitary transformation. The details of calculations for LBF and MBF can be found in Ref. [9] and Ref. [10], respectively.

\section{Numerical results and discussion}

We have solved the BdG equations together with a suitable transformation that diagonalize $H^{\mathrm{B}}$. The calculations have been carried out on a $32 \times 32$ cluster for $k_{\mathrm{B}} T=0.05 t$, and $\lambda=2.0 t$. In the LBF approach we have taken $\mu=-0.4 t$, $V=0.5 t$ and $E_{\mathrm{B}}=0.1 t$, whereas for $\mathrm{MBF} \mu=-0.2 t, V=1.0 t, E_{\mathrm{B}}=0.7 t$ and $\omega_{\langle i, j\rangle}=-0.1 t$ have been assumed. The model parameters have been chosen in such a way that the average concentration of bosons is much smaller than unity.

We have started our investigations with a system containing a single impurity (see Fig. 1). Here, $\Delta_{\max }$ is the maximal value of $\Delta_{i}$ in a system. $\Delta_{\text {imp }}$ denotes $\Delta_{i}$ at site $i$ just below the impurity and $\Delta_{0}$ represents $\Delta_{i}$ away from the impurity. We have considered both positive and negative values of the impurity potential $U_{0}$. Since $U_{0}$ originates from the presence of negatively charged dopant oxygen atoms,
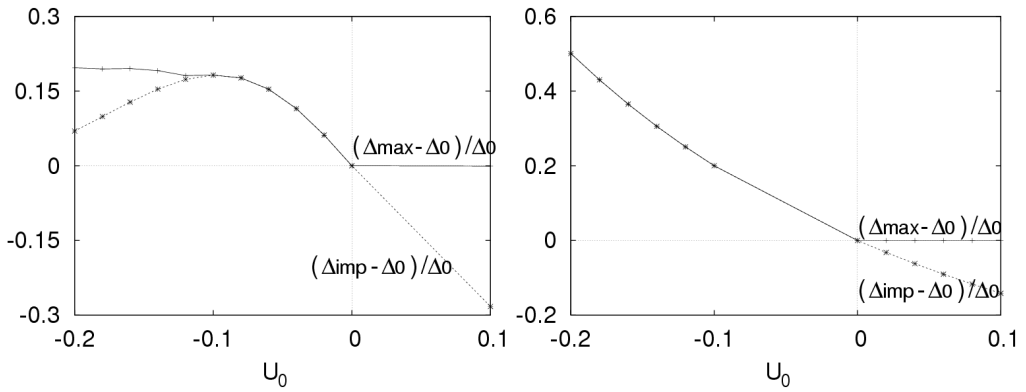

Fig. 1. Impurity potential dependence of the bosonic order parameter in LBF (left part) and MBF (right part) approaches calculated for a system with a single impurity.

$U_{0}>0$ implies that fermions and bosons represent electrons and electron pairs, respectively. On the other hand, for $U_{0}<0$ the boson-fermion model represents a system of itinerant holes and tightly bound hole pairs. As can be inferred from Fig. 1, LBF and MBF approaches lead to similar qualitative results for $U_{0}>0$ as well as for small negative $U_{0}$. In this regime the impurity potential $U_{0}>0$ $\left(U_{0}<0\right)$ is responsible for a reduction (enhancement) of the local concentration of bosons that, in turn, leads to a decrease (increase) in $\Delta_{\mathrm{imp}}$. The experimentally observed enhancement of the superconducting gap in the vicinity of impurities occurs only for $U_{0}<0$, i.e., only for bosons which represent pairs of tightly bound holes. It would be important to verify whether this observation holds true 
also beyond the mean-field approximation. Importance of the hard-core effects in the bosonic subsystem becomes visible already for moderate negative $U_{0}$. In the MBF approach $\Delta_{\text {imp }}=\Delta_{\max }$ and $\Delta_{\text {imp }}$ increases monotonically when $U_{0}$ decreases. In the LBF approach $\Delta_{\mathrm{imp}}$ is maximal for such value of $U_{0}$ that the concentration of bosons in the vicinity of impurity is close to $1 / 2$. For stronger attraction $\Delta_{\text {imp }}$ decreases, whereas the maximal value of $\Delta_{i}$ shifts away from the impurity. Contrary to MBF $\Delta_{\max }$ saturates for sufficiently strong negative $U_{0}$.

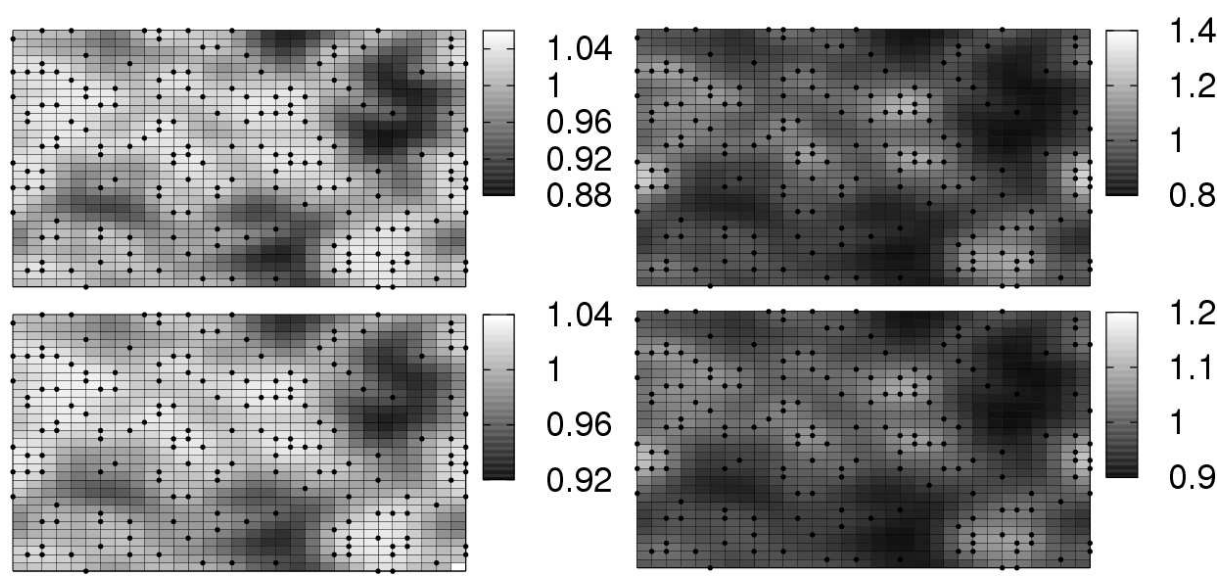

Fig. 2. Spatial variation of fermionic (top parts) and bosonic (bottom parts) order parameters normalized to their average values. Left and right parts represent results obtained for LBF and MBF, respectively. The impurities are marked as dots.
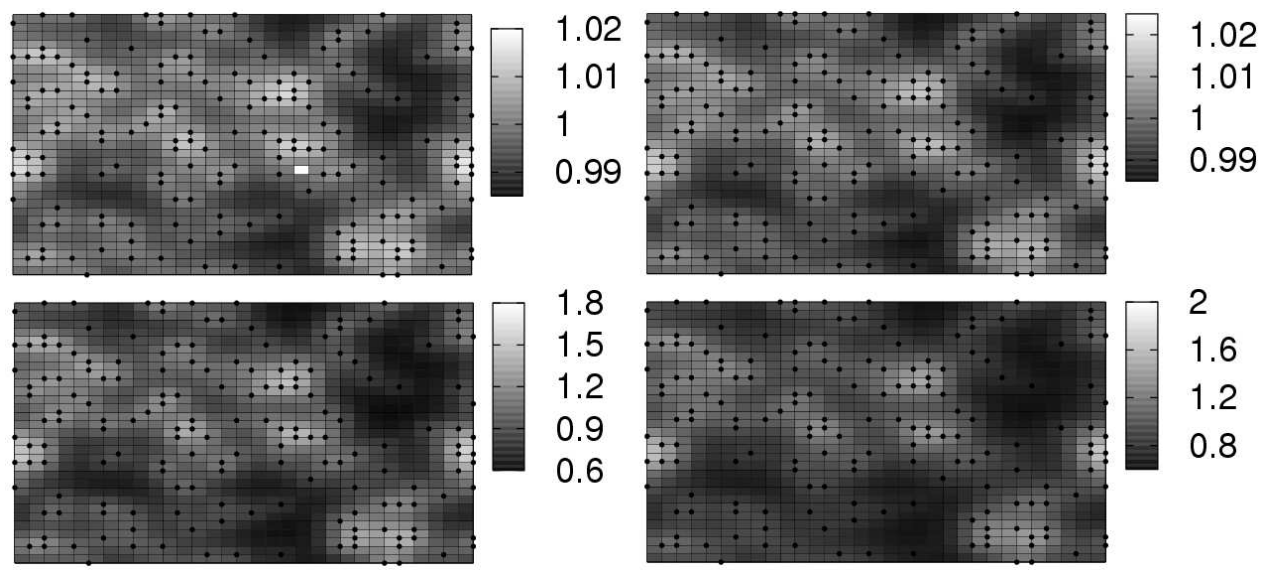

Fig. 3. Spatial variation of fermionic (top parts) and bosonic (bottom parts) concentrations normalized to their average values. Left and right parts represent results obtained for $\mathrm{LBF}$ and $\mathrm{MBF}$, respectively. 
Next, we have considered a more realistic situation. Namely, we have analyzed the superconducting gap for a system with 170 randomly distributed impurities, which gives concentration 16\%. Results presented in Figs. 2 and 3 have been obtained for $U_{0}=-0.025 t$. One can see that spatial distribution of fermionic and bosonic order parameters is qualitatively the same. Although, the impurities-induced enhancement of $\Delta_{i}$ is more significant in MBF, the differences between $\mathrm{LBF}$ and MBF are only of quantitative character. Let us note that already very weak impurity potential may be responsible for significant modulation of the order parameters. This modulation is accompanied by a strongly inhomogeneous distribution of bosons but almost homogeneous distribution of fermions. It strongly contrasts with our recent results obtained for purely fermionic model [11]. In this approach, explanation of the experimental results requires much stronger dopant's electrostatic potential, that causes stronger inhomogeneity in the distribution of fermions. It is the most important conclusion following from the presented analysis.

\section{Acknowledgments}

This work has been supported by the Polish Ministry of Education and Science under grant No. 1 P03B 07130.

\section{References}

[1] S.H. Pan, J.P. O'Neal, R.L. Badzey, C. Chamon, H. Ding, J.R. Engelbrecht, Z. Wang, H. Eisaki, S. Uchida, A.K. Guptak, K.W. Ng, E.W. Hudson, K.M. Lang, J.C. Davis, Nature 413, 282 (2001).

[2] K.M. Lang, V. Madhavan, J.E. Hoffmann, E.W. Hudson, H. Eisaki, S. Uchida, J.C. Davis, Nature 415, 412 (2002).

[3] K. McElroy, J. Lee, J.A. Slezak, D.H. Lee, H. Eisaki, S. Uchida, J.C. Davis, Science 309, 1048 (2005).

[4] J. Ranninger, S. Robaszkiewicz, Physica B 135, 468 (1985).

[5] R. Micnas, J. Ranninger, S. Robaszkiewicz, Rev. Mod. Phys. 62, 113 (1990).

[6] R. Micnas, Phys. Rev. B 76, 184507 (2007).

[7] T. Domański, M.M. Maśka, M. Mierzejewski, Phys. Rev. B 63, 144502 (2001).

[8] T. Domański, K.I. Wysokiński, Phys. Rev. B 66, 064517 (2002).

[9] M. Mierzejewski, Ż. Śledź, Phys. Status Solidi B 242, 449 (2005).

[10] ̇̇. Śledź, M. Mierzejewski, Physica B 378-380, 426 (2006).

[11] M.M. Maśka, Ż. Śledź, K. Czajka, M. Mierzejewski, Phys. Rev. Lett. 99, 147006 (2007). 